\title{
Finite-Time Convergence Stability of Lur'e Discrete-Time Systems
}

\author{
Boutheina SFAIHI, Sirine FEKIH, Mohamed BENREJEB \\ Université de Tunis El Manar, Ecole Nationale d'Ingénieurs de Tunis, \\ Laboratoire de Recherche en Automatique (LARA), \\ B.P. 37, 1002 Tunis, Le Belvédère, Tunisia. \\ Boutheina.sfaihi@isetr.rnu.tn; Sirine.fekih@enit.rnu.tn; Mohamed.benrejeb@enit.rnu.tn
}

\begin{abstract}
In this paper, a constructive design methodology for Finite-Time Stability (FTS) of a class of nonlinear discrete-time systems is proposed. A dead-beat controller that can achieve $n$-finite-time stability is constructed. Furthermore, stability conditions, based on the use of Borne and Gentina practical stability criterion and matrices in the Benrejeb arrow form, are synthesized. Similarity between transient behaviors of dead-beat controlled linearized and nonlinear third order systems are shown and concluding remarks are formulated.
\end{abstract}

Keywords: Discrete Lur'e Postnikov systems; Finite-time stability; Dead-beat control; Vector norm; Benrejeb arrow form matrix.

\section{Introduction}

Finite Time Stability (FTS) was introduced in the control literature in the Sixties in [14, 35]. Since that, FTS has become one of the most fundamental and challenging problems of the nonlinear control. Finite-time stability (or short-time stability) is a much stronger requirement than classical asymptotic stability. It requires that every solution trajectory of the studied system reaches the origin in a finite time, called settling time [9]. Many approaches were proposed in the literature to solve FTS of nonlinear continuous systems. In [9], a solid development for finite-time stability theory of non-Lipschitzian systems is provided and sensitivity of finite-time-stable systems to perturbations is investigated. Some necessary and sufficient conditions for FTS of systems with the uniqueness of solutions in forward time are given in [25]. In [33], finite-time stability and finite-time boundedness sufficient conditions for systems with polynomial vector fields are provided and computational method checking developed conditions introduced.

Moreover, finite-time control and stabilization techniques have developed, in the last decades, an increasing attention in nonlinear control systems theory [17]. Considered first, in the literature of time-optimal control, one of the main advantages of the finite-time control strategy, is its ability to force a control system to reach a specified target in finite time. By consequence, various theoretical control techniques were developed for different classes of nonlinear continuous systems. In this context, feedback finite-time stabilization controllers of double integrators systems was considered in [8] and [19], perturbed double integrator systems in [32], chain of integrators systems in [27], higher-order controllable systems in [18], systems with parametric and dynamic uncertainties in [20], large-scale interconnected dynamical systems in [26], stochastic systems in [36] and nonlinear systems which can be represented by affine fuzzy system in [23].

Although the method has potential application to practical discrete-time processes, the study of feedback finite-time stabilizing controllers of discrete-time systems is quite underdeveloped and most of the results in the literature are focused on the linear case. Actually, feedback finite-time stabilizing controllers are synthesized for linear perturbed systems in [2], for uncertain linear perturbed systems in [38], for linear time-varying systems in [1] and, for linear systems with time-varying delay in [37]. In the last few years, other contributions on finite-time stabilization of nonlinear discretetime systems have been introduced in [23], for systems which can be represented by affine fuzzy system, and in [15], for a class of lowertriangular nonlinear systems.

In this work, the finite-time stabilization problem for the class of discrete-time nonlinear Lur'e Postnikov systems [24] is considered. A procedure showing how to develop a compensator ensuring the system trajectories convergence to zero, in finite sampling time, is introduced. Transient behaviours of the 
controlled nonlinear and linearized systems are then discussed.

This paper is organized as follows. In Section 2 , the class of the discrete-time Lur'e Postnikov-type systems is introduced and the problem formally stated. In Section 3, the existence conditions of a state feedback deadbeat controller guaranteeing $n-\mathrm{FTS}$ convergence of the $n$-order linearized discretetime system, is provided. In Section 4, sufficient stability conditions for Lur'e system are developed via the Borne and Gentina stability criterion and the Benrejeb arrow form matrix, and the case of a third order Lur'e system discussed. Concluding remarks are provided in Section 5.

\section{System Description and Problem Statement}

Let consider the class of Lur'e discrete-time system $(S)$ of Figure 1, where $r \in \mathfrak{R}$ denotes the reference input, $\varepsilon\left(k T_{s}\right) \in \Re$ the control error at the instant $k T_{s}$ denoted $\varepsilon_{k}, T_{s}$ the sampling time and $y \in \mathfrak{R}$ the output of the closed loop system. $f\left(\varepsilon_{k}\right): \Re \rightarrow \Re$ represents a memoryless nonlinear valued function, $B_{0}$ a zero order holder and $D(s)$ and $N(s)$ polynomials defined by

$$
\begin{aligned}
& D(s)=a_{0}+a_{1} s+\cdots+a_{n} s^{n} \\
& N(s)=\lambda_{0}+\lambda_{1} s+\cdots+\lambda_{n-1} s^{n-1}
\end{aligned}
$$

Where the coefficients $a_{i}$ and $\lambda_{j}$ for $\forall i=0,1, \ldots, n$ and $\forall j=0,1, \ldots, n-1$, are constant parameters such that $a_{n} \neq 0$ and $N(s)$ characterizing the nonlinear system state feedback compensator.

The introduced nonlinear discrete time system $(S)$ can be described by the following nonlinear recursive scalar equation

$$
S: \varepsilon_{k+n}+\sum_{i=1}^{n} a_{i}^{*}\left(\varepsilon_{k+n-1}\right) \varepsilon_{k+n-1}=r_{k+n}+\sum_{i=1}^{n} b_{i} r_{k+n-1}
$$

which, for $r=0$, becomes

$$
S: \varepsilon_{k+n}+\sum_{i=1}^{n} a_{i}^{*}\left(\varepsilon_{k+n-1}\right) \varepsilon_{k+n-1}=0
$$

By noting $x_{i, k}$ the $i^{\text {th }}$ component of the state vector $x_{k} \in \mathfrak{R}^{n}$ at instant $k T_{s}$ and choosing $x_{n, k}=\varepsilon_{k} \quad$ and $\quad x_{i, k+1}, \quad \forall i=1, \ldots, p \quad$ and $\forall p=2, \ldots, n$ such that

$$
\left\{\begin{array}{l}
x_{1, k+1}=-a_{n}^{*}\left(\varepsilon_{k}\right) x_{n, k} \\
x_{p, k+1}=-a_{n-p+1}^{*}\left(\varepsilon_{k}\right) x_{n, k}+x_{p-1, k}
\end{array}\right.
$$

the corresponding state space studied system description, can be written as

$$
S: x_{k+1}=A_{B F}\left(\varepsilon_{k}\right) x_{k}
$$

where the instantaneous characteristic matrix $A_{B F}\left(\varepsilon_{k}\right)$ is expressed in the Frobenius form as

$$
A_{B F}\left(\varepsilon_{k}\right)=\left[\begin{array}{ccccc}
0 & \cdots & \cdots & 0 & -a_{n}^{*}\left(\varepsilon_{k}\right) \\
1 & \ddots & & \vdots & -a_{n-1}^{*}\left(\varepsilon_{k}\right) \\
0 & \ddots & \ddots & \vdots & \vdots \\
\vdots & \ddots & \ddots & 0 & \vdots \\
0 & \cdots & 0 & 1 & -a_{1}^{*}\left(\varepsilon_{k}\right)
\end{array}\right]
$$

The main aims of this paper is, firstly, to find a dead-beat controller ensuring the FTS convergence of the studied system (4) or (6) (7), with a transient behavior elimination in a finite sampling time, and, secondly, to compare the finite-time stability efficiencies of the controlled studied nonlinear system with those of the corresponding linearized one.

\section{Transient Behavior Elimination of Linear Systems}

In classical control engineering [3, 34], deadbeat control $[13,16,29,31]$ is considered as an advanced control design technique, developed in the context of finite time stabilization and finite settling time, which aims to perfectly tracking a step reference in a finite number of sampling periods.

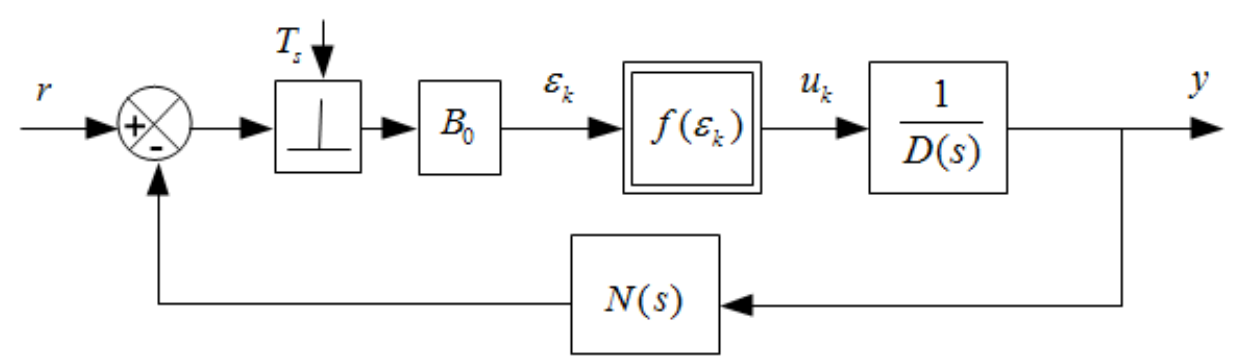

Figure 1. Lur'e discrete-time system 
Definition 1. [13] A stabilizing controller of $(S)$ is said to be a dead-beat controller if the tracking error $\varepsilon\left(k T_{s}\right)$ settles down to zero in a finite number of steps $k=N_{d}$ and $\varepsilon\left(k T_{s}\right)=0$, $\forall k \geq N_{d}$, where $T_{s}$ indicates the sampling period and $N_{d}$ the settling step.

In this section, we provide a methodology to design dead-beat controllers that achieve good transient performance of the linear discretetime system defined by (3) or by (6) - (7), when the nonlinearity $f\left(\varepsilon_{k}\right)$ is considered constant and equal to $K$. The main issue to solve this problem, is how to choose the controller parameters $\lambda_{i}, i=0,1, \ldots, n-1$ of the polynomial $N(s)$ which guarantee a transient response elimination in $n$ sampling time, where $n T_{s}$ constitutes the shortest possible settling time of a linear discrete-time control system for a given value of $T_{s}$ [31].

\subsection{Transient elimination in $\mathbf{n}$ steps}

Let consider the Lur'e system of Figure 1 where the nonlinearity $f\left(\varepsilon_{k}\right)$ is considered constant and equal to $K$. For $r=0$, the free system is described by the following equation

$$
\varepsilon_{k+n}+\sum_{i=1}^{n} a_{i}^{*} \varepsilon_{k+n-1}=0
$$

such that $a_{1}^{*}$ is a linear relation with the controller gains $\lambda_{j}, \quad \forall j=0,1, \ldots, n-1$

$$
a_{i}^{*}=b_{i}+K \sum_{j=0}^{n-1} a_{j}^{i} \lambda_{j}, \quad \forall i=1, \ldots, n
$$

The nth linear discrete-time system (8) is said to be FTC stable in $\mathrm{n}$ sampling time, if the gains $\lambda_{i}, \forall j=0,1, \ldots, n-1$ are synthesized, such that $\varepsilon_{k+n}$ settles down to zero in $\mathrm{n}$ steps, that's equivalent to setting $[10,29,31]$

$$
a_{i}^{*} \equiv 0 \quad \forall i=1,2, \ldots, n
$$

By solving the system equations (8) it comes the parameters of the state feedback control law characterized by $N(s)$ which guarantees the $n-$ time convergence to zero of the state variables.

\subsection{Case of a third order system}

\subsubsection{System description and analysis}

To show the interest of the proposed approach, let consider the third order linear closed loop discrete-time system $\left(S_{L}\right)$ such that $f()=$.$K and$

$$
\begin{aligned}
& D(s)=s\left(1+\tau_{1} s\right)\left(1+\tau_{2} s\right) \\
& N(s) \lambda_{0}+\lambda_{1} s+\lambda_{2} s
\end{aligned}
$$

Where $\tau_{1}, \tau_{2}, \lambda_{0}, \lambda_{1}$ and $\lambda_{2}$ are constant parameters. The system is described by

$$
S_{L}: \varepsilon_{k+3}+a_{1}^{*} \varepsilon_{k+2}+a_{2}^{*} \varepsilon_{k+1}+a_{3}^{*} \varepsilon_{k}=0
$$

Expressions of the coefficients $a_{3}^{*}, a_{2}^{*}$ and $a_{1}^{*}$ are given, respectively by (14), (15) and (16) for $f=K$.

$$
\begin{aligned}
& a_{3}^{*}=\left(\tau_{2}-\tau_{1}\right)^{-1} \times e^{\frac{-T_{s}\left(\tau_{1}-\tau_{2}\right)}{\tau_{1} \tau_{2}}} \times \\
& \times\left(\begin{array}{l}
\tau_{1}-\tau_{2}-f \lambda_{0}\left(\tau_{1}^{2}-\tau_{2}^{2}-\tau_{1}^{2} d_{1}^{-1}+\tau_{2}^{2} d_{2}^{-1}\right)+ \\
+f \lambda_{1}\left(\tau_{1}-\tau_{2}-\tau_{1} d_{1}^{-1}+\tau_{2} d_{2}^{-1}\right)+ \\
+f \lambda_{2}\left(d_{1}^{-1}-d_{2}^{-1}\right)-T_{s} f \lambda_{0}\left(\tau_{1}-\tau_{2}\right)
\end{array}\right) \\
& a_{2}^{*} \mp f \times\left(\left(d_{1}^{-1} d_{2}^{-1}\right)\left(\tau_{2}-\tau_{1}\right)\right)^{-1} \\
& \lambda_{0} \tau_{1}^{2}\left(1-d_{1}^{-1}+d_{2}^{-1}-d_{1}^{-1} d_{2}^{-1}\right) \\
& -2 \lambda_{2} d_{1}^{-1}-\lambda_{0} \tau_{2}^{2}\left(\begin{array}{l}
1+d_{1}^{-1}-d_{2}^{-1} \\
-d_{1}^{-1} d_{2}^{-1}
\end{array}\right) \\
& \times+2 \lambda_{2} d_{2}^{-1}+\tau_{1}\left(\lambda_{1}\left(\begin{array}{l}
d_{1}^{-1}-d_{2}^{-1} \\
+d_{1}^{-1} d_{2}^{-1}-1
\end{array}\right)\right)
\end{aligned}
$$

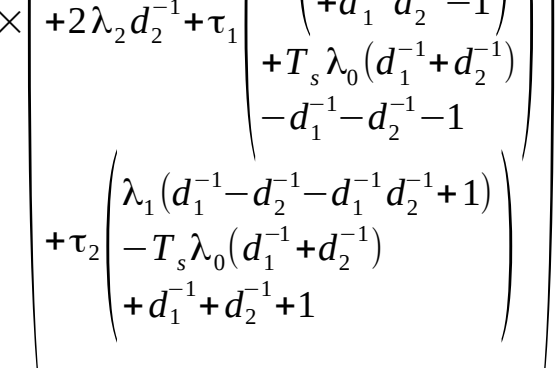

$$
\begin{aligned}
& a_{1}^{*} \bar{\mp}\left(d_{1}^{-1} d_{2}^{-1}\left(\tau_{2}-\tau_{1}\right)\right)^{-1} \times \\
& \mid \begin{array}{l}
f \lambda_{0}\left(\begin{array}{l}
d_{2}^{-1}\left(d_{1}^{-1}-d_{2}^{-1}\right) \tau_{1}^{2}+ \\
+d_{1}^{-1}\left(1-d_{2}^{-1}\right) \tau_{2}^{2}
\end{array}\right)+ \\
+\tau_{1}\left(\begin{array}{l}
d_{1}^{-1}+d_{2}^{-1}+d_{1}^{-1} d_{2}^{-1}+ \\
+f \lambda_{1} d_{2}^{-1}\left(1-d_{1}^{-1}\right)- \\
-T_{s} f \lambda_{0} d_{1}^{-1} d_{2}^{-1} \\
d_{2}^{-1}+d_{1}^{-1}+d_{1}^{-1} d_{2}^{-1}+ \\
+f \lambda_{1} d_{1}^{-1}\left(1-d_{2}^{-1}\right)- \\
-T_{s} f \lambda_{0} d_{1}^{-1} d_{2}^{-1}
\end{array}\right)+ \\
+f \lambda_{2}\left(d_{1}^{-1}-d_{2}^{-1}\right)
\end{array}
\end{aligned}
$$

with $d_{1}^{-1}=e^{\frac{T_{s}}{\tau_{1}}}$ and $d_{2}^{-1}=e^{\frac{T_{s}}{\tau_{2}}}$.

A state space description $\left(S_{L}\right)$ associated to the linear system (8) with (14) - (16) and 
developed in the Fobenius canonial form, can be introduced as

$S_{L}:\left[\begin{array}{c}z_{k+1} \\ w_{k+1} \\ \varepsilon_{k+1}\end{array}\right]=\left[\begin{array}{ccc}0 & 0 & -a_{3}^{*} \\ 1 & 0 & -a_{2}^{*} \\ 0 & 1 & -a_{1}^{*}\end{array}\right]\left[\begin{array}{c}z_{k} \\ w_{k} \\ \varepsilon_{k}\end{array}\right]$

By applying (10), setting $a_{i}^{*}=0, \forall i=1,2,3$ and choosing $\lambda_{0}=1$, the transient behaviour of the closed loop system (13) with (14) - (16) or (17) is eliminated in three sampling periods for $K, \lambda_{1}$ and $\lambda_{2}$ defined, respectively, by

$$
\begin{aligned}
& K=\left(T_{s}\left(1-d_{1}\right)\left(1-d_{2}\right)\right)^{-1} \\
& \lambda_{1}=\tau_{1}+\tau_{2}-T_{s} \frac{\left(d_{1}+d_{2}-d_{1} d_{2}\right)^{2}-d_{1} d_{2}}{\left(1-d_{1}\right)\left(1-d_{2}\right)} \\
& \lambda_{2}=\tau_{1} \tau_{2}-T_{s} \frac{\tau_{1} d_{2}^{3}\left(1-d_{1}\right)^{2}-\tau_{2} d_{1}^{3}\left(1-d_{2}\right)^{2}}{\left(d_{1}-d_{2}\right)\left(1-d_{1}\right)\left(1-d_{2}\right)}
\end{aligned}
$$

with $d_{1}=e^{\frac{T_{s}}{\tau_{1}}}$ and $d_{2}=e^{\frac{T_{s}}{\tau_{2}}}$.

Equations (18) - (20) characterize solutions to the FTS problem for the third order nonlinear plant $\left(S_{L}\right)$.

\subsubsection{Simulation results}

The plant $\left(S_{L}\right)$ is used for simulations where the constant times system are chosen to be $\tau_{1}=0,1 \mathrm{~s}$ and $\tau_{2}=0,05 \mathrm{~s}$, the sampling period $T_{\mathrm{s}}=0,2 \mathrm{~s}$ and the initial conditions $x_{1}(0)=0,1, \quad x_{2}(0)=0,011 \quad$ and $x_{3}(0)=-0,073$. Satisfaction of the FTS conditions (18) - (20) is accomplished by making the static gain $\mathrm{K}$ and the dead-beat controller gains $\lambda_{1}$ and $\lambda_{2}$, respectively equal to

$$
\begin{aligned}
& K=K_{F T C S}=5,89 \\
& \lambda_{1}=0,14 \\
& \lambda_{2}=0,48 \times 10^{-2}
\end{aligned}
$$

The system with the given controller parameters is asymptotically stable for the sector domain $D_{1}$, defined by

$$
D_{1}=\left\{K \mid 0,2 \times 10^{-3}<K<10,35\right\}
$$

as shown in Figsures 2, 3 and 4. With the same control law (12) with (22) - (23), the system dynamics, for $K=K_{F T C S}$, are presented in Figure 2 and, for $K=4$, in Figure 4. Simulations results for $K=K_{F T C S}$ highlight the three-sampling-time stability convergence $(0,6 s)$ of $\left(S_{L}\right)$, as depicted in Figure 2. By varying $K$ in $D_{1}$, the system transient behaviour becomes more important than for the particular case $K=K_{\text {FTCS }}$. Comparing results of Figure 2 with those of Figure 4, one can note that the stability convergence is obtained for a number of sampling times $m, m>3$.

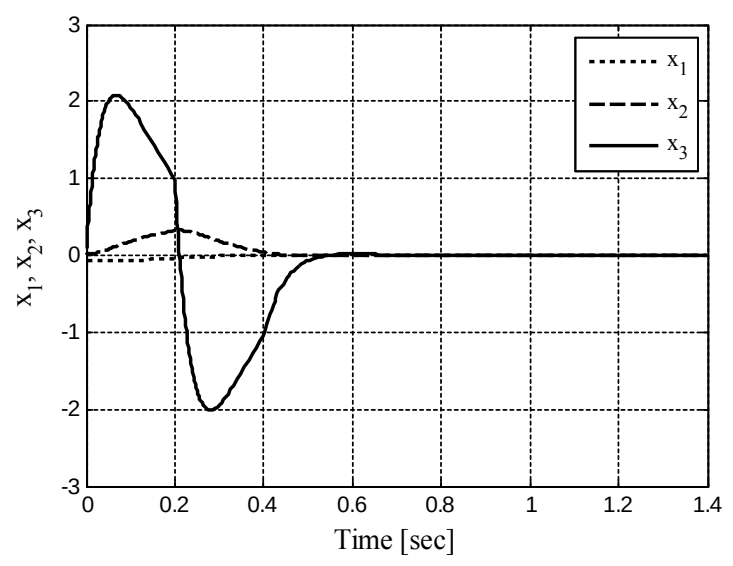

Figure 2. System dynamics for: $K=K_{F T C S}$

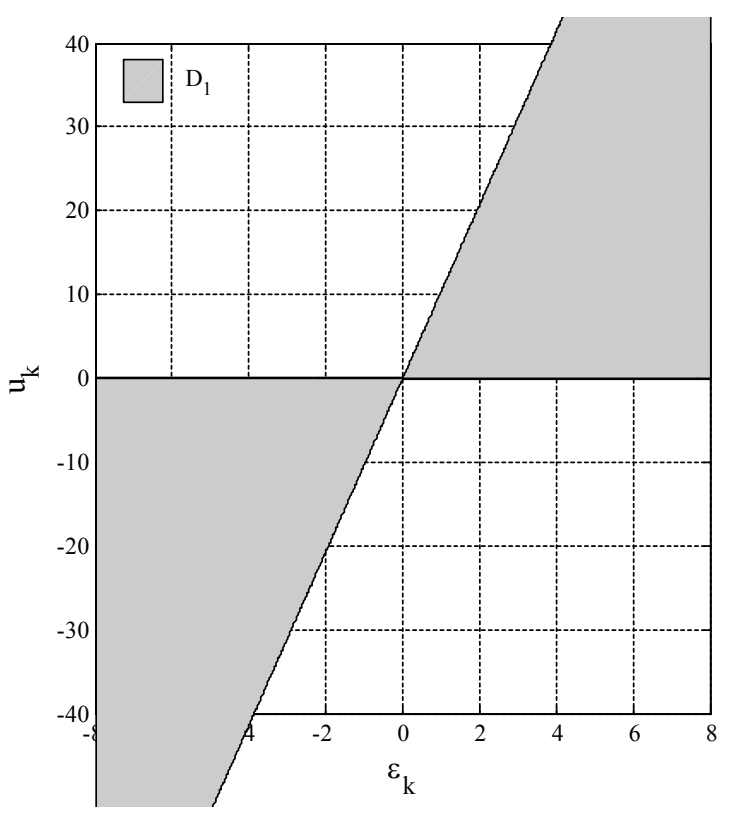

Figure 3. Linear system stability domain

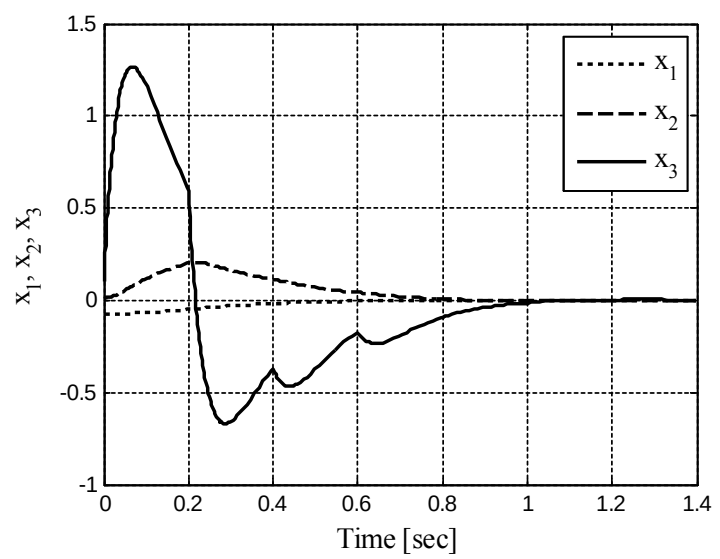

Figure 4. System dynamics for $K=4$ 


\section{Stability Convergence Conditions for Nonlinear Systems}

Most of stability results of nonlinear Lur'e Postnikov systems focused on frequency analysis: absolute stability [24], Popov criterion [28] and circle criterion [21]. In this section, stability conditions for the Lur'e Postnikov systems (6) - (7) are developed using the Borne and Gentina stability criterion [10-12] (Appendix) and the Benrejeb arrow form matrix [4-7]. Moreover, a stabilizing dead-beat controller for the nonlinear studied system (4) or (6) - (7) exploiting the controller parameters of the linearized system (13) with (14) - (16) or (17) is proposed.

\subsection{Lur'e system stability analysis}

Let $(S)$ be a Lur'e-type system of the form (4) or (6)-(7), $a_{j}, \forall j=1,2, \ldots, n-1, a_{i} \neq a_{j}, \forall i \neq j$ be distinct arbitrary constant parameters and $\beta_{j}$, $\gamma_{j}$ and $\gamma_{n}$ be parameters expressed respectively by [4-7].

$$
\begin{aligned}
& \beta_{j}=\prod_{\substack{k=1 \\
k \neq j}}^{n-1}\left(\alpha_{j}-\alpha_{k}\right)^{-1}, \forall j=1, \ldots, n-1 \\
& \gamma_{j}(.)=-P_{A_{B F}}\left(, \alpha_{j}\right), \forall j=1, \ldots, n-1 \\
& \gamma_{n}(.)=-a_{1}(.)-\sum_{i=1}^{n-1} \alpha_{i}
\end{aligned}
$$

$P_{A_{B F}}(., \lambda)$ is the instantaneous characteristic polynomial such that

$$
P_{A_{B F}}(., \lambda)=\lambda^{n}+\sum_{i=1}^{n} a_{i}(.) \lambda^{n-1}
$$

By applying the Borne and Gentina practical stability criterion $[11,12]$ to the discrete Lur'e type system (4) or (6) - (7) characterized by the Benrejeb arrow form matrix [4-7], it comes the following theorem [30].

Theorem. The Lur'e Postnikov discrete-time system $(S)$ (4) or (6) -(7) is asymptotically stable, if there exists constant parameters $\alpha_{i}$, $\forall i, j=1,2, \ldots, n-1, \alpha_{i} \neq \alpha_{j}, \forall i \neq j \quad$ and $\varepsilon>0$ such that $\forall x_{k} \in D \subset \Re^{n}$, the following conditions are satisfied

$$
\begin{aligned}
& \left|\alpha_{i}\right|<1, \forall i=1, \ldots, n-1 \\
& 1-\left|\gamma_{n}(.)\right|-\sum_{j=1}^{n-1}\left|\gamma_{j}(.)\right|\left|\beta_{j}\right|\left(1-\left|\alpha_{j}\right|\right)^{-1} \geq \varepsilon
\end{aligned}
$$

If $D=\mathfrak{R}^{n}$, the stability is global.

Proof. Let consider the Lur'e type system $(S)$ of the state representation form (6). A change of coordinate defined by $y_{k}=T x_{k}$ with $y_{k} \in \mathfrak{R}^{n}$ and $T \in \mathfrak{R}^{n \times n}$ inversible, defined by

$$
T=\left[\begin{array}{ccccc}
0 & 0 & \cdots & 0 & 1 \\
1 & \alpha_{1} & \cdots & \alpha_{1}^{n-2} & \alpha_{1}^{n-1} \\
\vdots & \vdots & \cdots & \vdots & \vdots \\
1 & \alpha_{n-1} & \cdots & \alpha_{n-1}^{n-2} & \alpha_{n-1}^{n-1}
\end{array}\right]
$$

leads to the following state space description

$$
y_{k+1}=A_{F}(.) y_{k}
$$

where $A_{F}($.$) is an arrow form matrix given by$

$$
A_{F}(.)=\left[\begin{array}{cccc}
\gamma_{n}(.) & \beta_{1} & \cdots & \beta_{n-1} \\
\gamma_{1}(.) & \alpha_{1} & & \\
\vdots & & \ddots & \\
\gamma_{n-1}(.) & & & \alpha_{n-1}
\end{array}\right]
$$

and $\beta_{i}, \gamma_{j}, \gamma_{n}$ and $\alpha_{i}, \forall i=1,2, \cdots, n-1$ defined respectively in relations (25) - (27) with (28). A pseudo-overvaluing matrix $M\left(A_{F}().\right)$ of (32), corresponding to the use of the vector norm $p\left(y_{k}\right)=\left[\left|y_{1, k}\right|,\left|y_{2, k}\right|, \cdots,\left|y_{n, k}\right|\right]^{T} \quad$ with $y_{k}=\left[y_{1, k}, y_{2, k}, \cdots, y_{n, k}\right]^{T}, \quad$ for the stability study, such that [12]

$$
p\left(y_{k+1}\right) \leq M\left(A_{F}(.)\right) p\left(y_{k}\right)
$$

leads to the following comparison system

$$
\begin{aligned}
& z_{k+1}=M\left(A_{F}(.)\right) z_{k} \\
& M\left(A_{F}\right)=\left[\begin{array}{cccc}
\left|\gamma_{n}(.)\right| & \left|\beta_{1}\right| & \cdots & \left|\beta_{n-1}\right| \\
\left|\gamma_{1}(.)\right| & \left|\alpha_{1}\right| & & \\
\vdots & & \ddots & \\
\left|\gamma_{n-1}(.)\right| & & & \left|\alpha_{n-1}\right|
\end{array}\right]
\end{aligned}
$$

with $M\left(A_{F}().\right) \in \mathfrak{R}^{n \times n}, z_{k} \in \Re^{n}$ and $z_{0}=p\left(y_{0}\right)$. Since the nonlinearities of the comparison nonlinear system (35) are isolated in one row of $M\left(A_{F}().\right)$, stability conditions are, then, deduced by applying Borne and Gentina stability criterion

$$
\begin{array}{r}
\left(I_{n}-M\left(A_{F}(.)\right)\right)=\left(\begin{array}{cccc}
1 & 2 & \cdots & h \\
1 & 2 & \cdots & h
\end{array}\right)>0 \\
\forall h=1,2, \ldots, n
\end{array}
$$

This completes the proof.

Corollary. If there exists constant parameters $\varepsilon>0 \quad$ and $\quad \alpha_{j} \in \mathfrak{R}, 0<\alpha_{j}<1, \alpha_{j} \neq \alpha_{k}, \forall j \neq k$, 
$j, k=1, \cdots, n-1 \quad$ such that $\delta_{n}()>$.0 and $\gamma_{j}(.) \beta_{j}>0, \forall j=1, \ldots, n-1, \quad$ then, the nonlinear discrete time system $(S)(4)$ or $(6)-$ (7) is asymptotically stable if the following condition is satisfied

$$
\left.P_{s}(., \lambda)\right|_{\lambda=1} \geq \varepsilon
$$

Proof. If there exists constant parameters $\alpha_{j} \in \mathfrak{R}, \quad 0<\alpha_{j}<1, \quad \alpha_{j} \neq \alpha_{k}, \quad \forall j \neq k, \quad$ and $j, k=1, \ldots, n-1$ such that $\delta_{n}()>$.0 and $\gamma_{j}(.) \beta_{j}>0, \quad \forall j=1, \ldots, n-1, \quad$ then, the comparison system (35) - (36) is identical to the system itself. Linear Aizerman conjecture is, consequently, satisfied. In this case, by substituting (25) - (27), stability condition (30) of $(S)$ becomes

$$
\left\{\begin{array}{l}
1+a_{1}(.)+\sum_{p=1}^{n-1} \alpha_{p}+ \\
+\sum_{p=1}^{n-1} \frac{1}{1-\alpha_{p}}\left(\frac{\left(\lambda-\alpha_{p}\right) P_{s}(, . \lambda)}{Q(\lambda)}\right)_{\lambda=\alpha_{p}}
\end{array}\right\}>0
$$

where $Q(\lambda)$ is defined by

$$
Q(\lambda)=\prod_{p=1}^{n-1}\left(\lambda-\alpha_{p}\right)
$$

Now, observing that,

$$
\begin{aligned}
& \frac{P_{S}(., \lambda)}{Q(\lambda)}=\lambda+a_{1}(.)+\sum_{p=1}^{n-1} \alpha_{p}+ \\
& \quad+\sum_{p=1}^{n-1} \frac{1}{\lambda-\alpha_{p}}\left(\frac{\left(\lambda-\alpha_{p}\right) P_{S}(., \lambda)}{Q(\lambda)}\right)_{\lambda=\alpha_{p}}
\end{aligned}
$$

stability condition (39) is, then, equivalent to

$$
\left.\frac{P_{S}(., \lambda)}{Q(\lambda)}\right|_{\lambda=1}>0
$$

or equivalently to

$$
\left.P_{S}(., \lambda)\right|_{\lambda=1}>0
$$

That ends the corollary proof.

\subsection{Finite time stability: Case of a third order Lur'e system}

We reconsider the nonlinear Lur'e third order system presented in the previous section and introduced in Figure 1 with (11) and (12). The system can be described by the following recursive equation

$$
\begin{aligned}
S: \varepsilon_{k+3}+a_{1}^{*} & \left(\varepsilon_{k+2}\right) \varepsilon_{k+2}+ \\
& +a_{2}^{*}\left(\varepsilon_{k+1}\right) \varepsilon_{k+1}+a_{3}^{*}\left(\varepsilon_{k}\right) \varepsilon_{k}=0
\end{aligned}
$$

where the expressions of the parameters $a_{3}^{*}($. , $a_{2}^{*}($.$) and a_{3}^{*}($.$) depending on the$ nonlinearity $f$ are defined respectively by (14), (15) and (16). By using (5), it comes the state space representation

$$
S:\left[\begin{array}{c}
z_{k+1} \\
w_{k+1} \\
\varepsilon_{k+1}
\end{array}\right]=\left[\begin{array}{ccc}
0 & 0 & -a_{3}^{*}\left(\varepsilon_{k}\right) \\
1 & 0 & -a_{2}^{*}\left(\varepsilon_{k}\right) \\
0 & 1 & -a_{1}^{*}\left(\varepsilon_{k}\right)
\end{array}\right]\left[\begin{array}{c}
z_{k} \\
w_{k} \\
\varepsilon_{k}
\end{array}\right]
$$

The dead-beat control for the linearized model $\left(S_{L}\right)$ (13) will, in reality, be applied to the nonlinear model $(S)$ (44), which includes property modifications. The effects of this choice on the FTS property of the resulting feedback system are to be investigated. This investigation will be simplified by representing the nonlinearity around $u_{k}=K_{F T C S} \varepsilon_{k}$.

For the defined system constants time and the synthesized controller parameters $\left(\lambda_{0}, \lambda_{1}, \lambda_{2}\right)$, the state matrix $A_{B F}($.) of (45), expressed in the Frobenius form, is such that

$$
A_{B F}(.)=\left[\begin{array}{ccc}
0 & 0 & 0,24 \times 10^{-2}-0,50 \times 10^{-3} f\left(\varepsilon_{k}\right) \\
1 & 0 & -0,15+0,26 \times 10^{-1} f\left(\varepsilon_{k}\right) \\
0 & 1 & 1,15-0,19 f\left(\varepsilon_{k}\right)
\end{array}\right]
$$

Now, using the transformation (31) - (35) to (45) with $\alpha_{1}=0,14$ and $\alpha_{2}=0,02$ satisfying (29), the new state matrix of (44) or (45) - (46) is in the Benrejeb arrow form as following

$$
\begin{aligned}
& A_{F}(.)=\left[\begin{array}{c}
0,99-0,19 f\left(\varepsilon_{k}\right) \\
0,50 \times 10^{-3}-0,60 \times 10^{-3} f\left(\varepsilon_{k}\right) \\
-0,20 \times 10^{-3}-0,30 \times 10^{-4} f\left(\varepsilon_{k}\right)
\end{array}\right. \\
& \left.\begin{array}{cc}
8,33 & -8,33 \\
0,14 & 0 \\
0 & 0,02
\end{array}\right]
\end{aligned}
$$

Stability condition of $(S)$ characterized by (44) or (45) - (46), deduced from the developed Theorem, is then

$$
\left\{\begin{array}{l}
1-\left|0,99-0,19 f\left(\varepsilon_{k}\right)\right|- \\
-9.68 \times 10^{-3} \times\left|0,50-0,6 f\left(\varepsilon_{k}\right)\right|- \\
-8.5 \times 10^{-3} \times \mid-0,02-0.03 \times 10^{-1} f\left(\varepsilon_{k}\right)
\end{array}\right\}>0
$$

equivalent to

$$
D_{2}: 0,30 \times 10^{-3}<f(.)<9,88
$$

For purposes of comparison with the linearized system $\left(S_{L}\right)$ behaviour, let consider the nonlinear function $f\left(\varepsilon_{k}\right)=c_{1}\left(\varepsilon_{k}+c_{2} \sin \left(\varepsilon_{k}\right)^{p}\right)$ 
and study the influence of the constant parameters $c_{1}, c_{2}$ and $p$, on the elimination time of the transient. Three cases are introduced in Table 1.

Table 1. Parameters definition of $f\left(\varepsilon_{k}\right)$

\begin{tabular}{|l|c|c|c|}
\cline { 2 - 4 } \multicolumn{1}{c|}{} & $c_{1}$ & $c_{2}$ & $p$ \\
\hline Case 1 & 4 & 0.5 & 2 \\
\hline Case 2 & 5.89 & 0.5 & 2 \\
\hline Case 3 & 5.89 & 0.7 & 5 \\
\hline
\end{tabular}

The responses of the system, with the cases 1,2 and 3 of $f\left(\varepsilon_{k}\right)$, are shown for initial values of $x(0)=[0.1,0.011,-0.073]^{T}$ in, respectively, Figures 5, 7 and 9. As shown in cited Figures, $f\left(\varepsilon_{k}\right)$ is in the sector characterized by (48) for all considered cases. The corresponding system controller stabilizes the Lur'e system $(S)$ as illustrated in Figures 6, 8 and 10. For case 1, $(S)$ remains stable but looses the proper of three sampling period convergence to zero, as shown in Figure 6. For cases 2 and 3, the nonlinear functions vary around the line $u_{k}=K_{F T C S} \varepsilon_{k}$ and the transient behaviour is eliminated in three sampling periods, as shown in Figures 8 and 10. Figure 11 compares the response of the linearized and nonlinear (case 2) systems. When $u_{k}$ is around $K_{F T C S} \varepsilon_{k}$, the $\left(S_{L}\right)$ and $(S)$ responses are nearly identical. Else, the responses are significantly different, the system is FTS in $n+m$ step times with $m>0$.

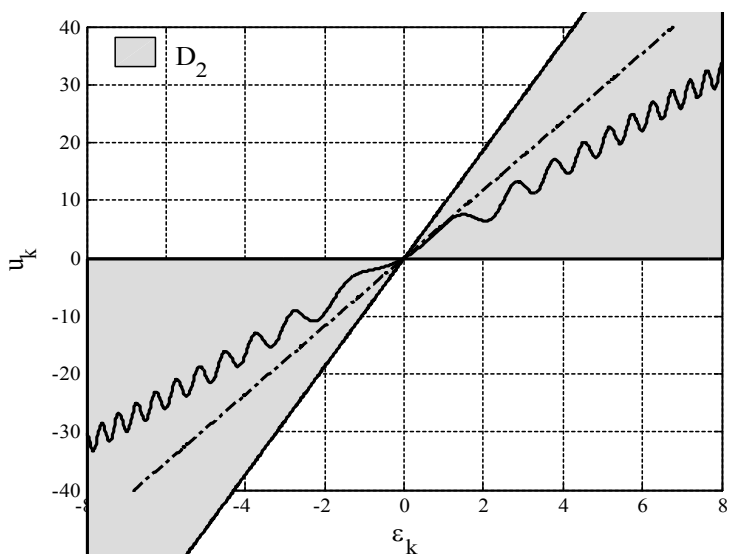

Figure 5. Nonlinear function - Case 1

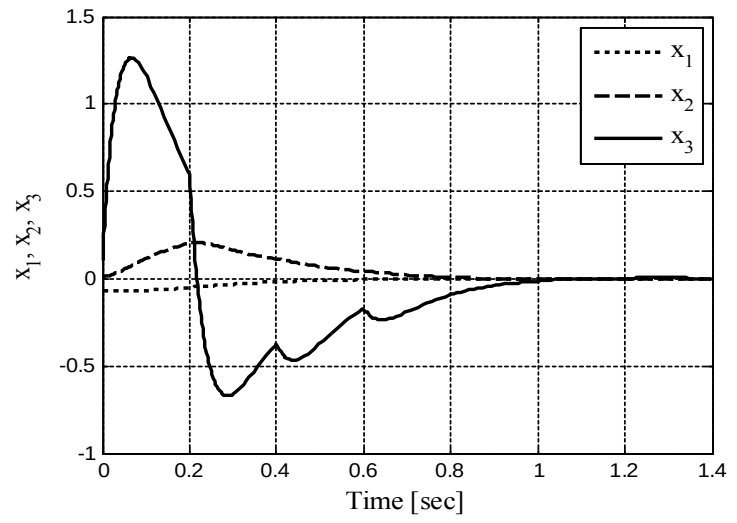

Figure 6. System dynamics - Case 1

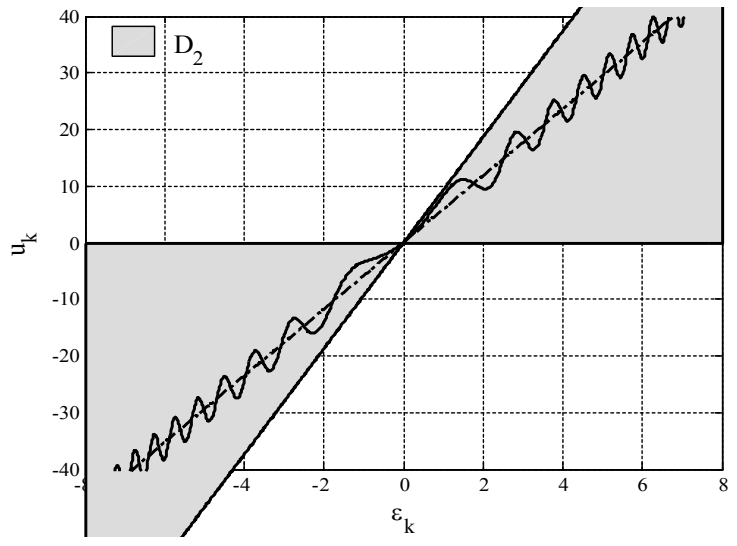

Figure7. Nonlinear function- Case 2

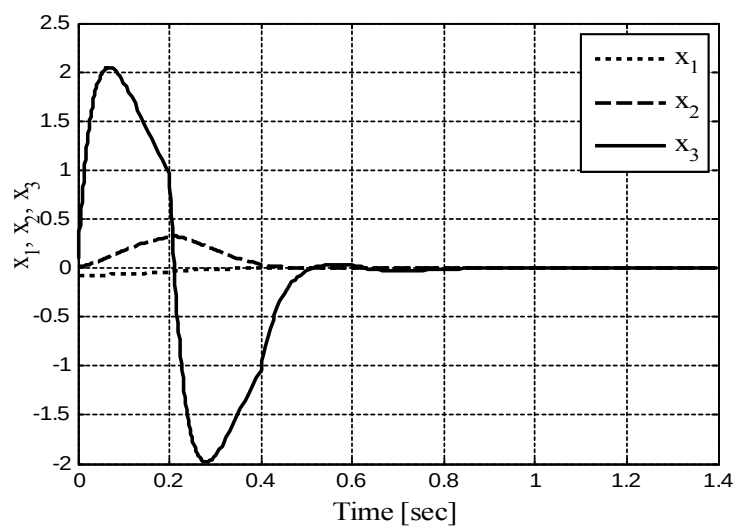

Figure 8. System dynamics - Case 2

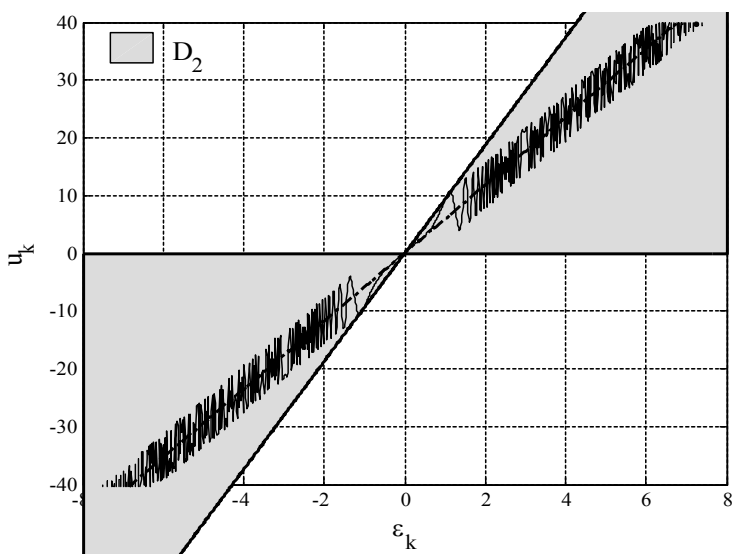

Figure 9. Nonlinear function - Case 3 


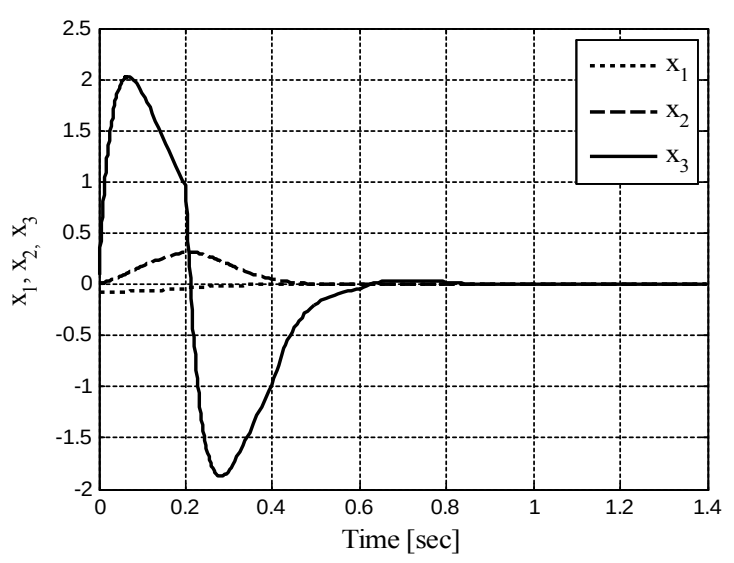

Figure 10. System dynamics - Case 3

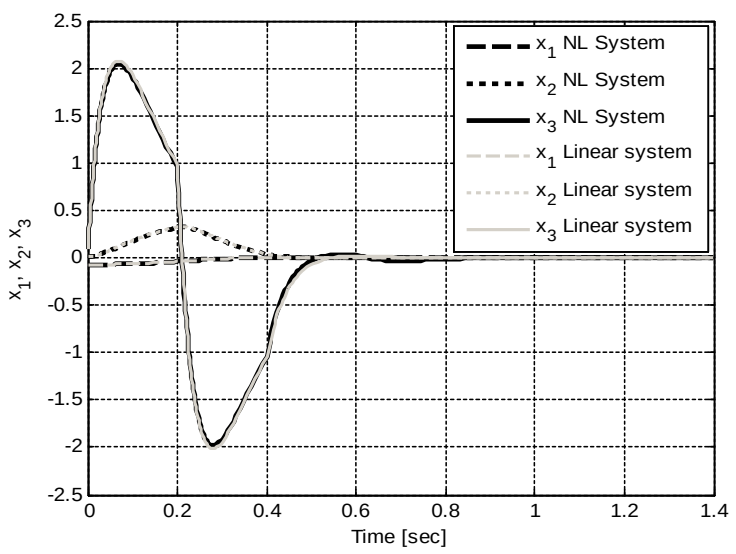

Figure 11. Linearized and nonlinear (Case2) System dynamics

\section{Conclusion}

The design approach considered in this paper, is based on specifying an $n$-FTS behavior for the Lur'e type nonlinear systems via the linearized system dead-beat controller. Although, this does not guarantee an $n$-FTS behavior in general case, linearization approaches have proved successful in many practical situations in literature. Validity of the proposed method was illustrated with success via an example of a third order Lur'e system.

\section{Appendix}

Definition 2. (Vector Norm) Let $E=\Re^{n}$ be a vector space and $E_{1}, E_{2}, \cdots, E_{k}$ subspaces of $E$ which verify: $e=E_{1} \cup E_{2} \cup \cdots \cup E_{k}$. Let $x \in E$ be an $n$ vector defined on $E$ with a projection in the subspace $E_{i}$ denoted by $x_{i}, x_{i}=P_{i} x$, where $P_{i}$ is a projection operator from $E$ into $E_{i}$. Let $p_{i}$ be a scalar norm $(i=1, \cdots, k)$ defined on the subspace $E_{i} \quad p_{i}(x): \mathfrak{R}^{n} \rightarrow \mathfrak{R}_{+}^{k} \quad$ and $p(x)$ denotes the vector norm of dimension $k$ with $p(x)=\left[\begin{array}{llll}p_{1}^{T}(x) & p_{2}^{T}(x) & \ldots & p_{k}^{T}(x)\end{array}\right]^{T}$.

Kotelyanski Lemma [22]. The real parts of the eigenvalues of matrix $A$, with non negative off diagonal elements, are less than a real number $\mu$ if and only if all those of matrix $M=\mu I_{n}-A$ are positive, with $I_{n}$ the $n$ identity matrix. When successive principal minors of matrix $(-A)$ are positive, Kotelyanski lemma permits to conclude on stability property of the system characterized by $A$.

Borne and Gentina practical stability criterion [11, 12]. Let consider the nonlinear discrete system

$z_{k+1}=A(.) z_{k}$

and the overvaluing matrix $M(A().) \in \mathfrak{R}^{n \times n}$ associated to the use of the vector norm $p_{z}$ defined by $p_{z}=\left[\left|z_{1, k}\right|,\left|z_{2, k}\right|, \ldots,\left|z_{n, k}\right|\right]^{T} \quad$ with $z_{k}=\left[z_{1, k}, z_{2, k}, \ldots, z_{n, k}\right]^{T}$ and such that

$$
M(A(.))=\left\{\left|a_{j, k}\right|\right\}, \quad \forall j, k=1, \cdots, n
$$

If the nonlinearities are isolated in either one row or one column of $M(A()$.$) , the verification$ of the Kotelyanski condition enables to conclude to the stability of the original system characterized by $A($.$) .$

The system (49) is asymptotically stable if all successive principal minors of $\left(I_{n}-M(A()).\right)$ are positive.

\section{REFERENCES}

1. F. AMATO, M. ARIOLA, C. COSENTINO, Finite-time Control of Discrete-time Linear Systems: Analysis and Design Conditions, Automatica, 46(5), 2010, pp. 919-924.

2. F. AMATO, M. ARIOLA, Finite-time Control of Discrete-time Linear Systems, IEEE Transactions on Automatic Control, 50(5), 2005, pp. 724-729.

3. AMRI, I., D. SOUDANI, M. BENREJEB, Delay Dependent Robust Exponential Stability Criterion for Perturbed and Uncertain Neutral Systems with Time Varying Delays, Studies in Informatics nd Control, 19(2), 2010, pp. 135-144.

4. BENREJEB, M., P. BORNE, On an Algebraic Stability Criterion for 
Nonlinear Processes. Interpretation in the Frequency Domain, in: Proceedings of the Measurement and Control International Symposium (MECO'78), Athens, 1978.

5. BENREJEB, M., P. BORNE, F. LAURENT, On an Application of the Arrow Form Representation in the Processes Analysis (in French), RAIRO Automatique, 16(2), 1982, pp. 133-146.

6. BENREJEB, M., A. SAKLY, K. BEN OTHMAN, P. BORNE, Choice of Conjunctive Operator of TSK Fuzzy Systems and Stability Domain Study, Mathematics and Computers in Simulation, 76(5-6), 2008, pp. 410-421.

7. BENREJEB, M., Stability Study of Two Level Hierarchical Nonlinear Systems, Plenary lecture in: The 12th IFAC Large Scale Systems Symposium: Theory and Applications (IFAC LSS 2010), Lille.

8. BHAT, S. P., D. S. BERNSTEIN, Continuous Finite-time Stabilization of the Translational and Rotational Double Integrators, IEEE Trans. on Automatic Control, vol. 43(5), 1998, pp. 678-682.

9. BHAT, S. P., D. S. BERNSTEIN, Finitetime Stability of Continuous Autonomous Systems, SIAM J. Ctrl. and Optimization, 38(3), 2000, pp. 751-766.

10. BORNE, P. Analyse des systèmes asservis échantillonnés à paramètres périodiques (Doctorat Thesis), Faculté de Sciences de l’Université de Lille, 1970.

11. BORNE, P., J. C. GENTINA, F. LAURENT, Stability Study of Large Scale Nonlinear Discrete Systems by Use Vector Norms, in: Proceedings of the IFAC Symposium on Large Scale Systems: Theory and Applications (IFAC LSS), Udine, 1976.

12. BORNE, P., J. P. RICHARD, N. E. RADHY, in: A. J. Fossard and D. Normand-Cyrot (Eds), Stability, Stabilization, Regulation using Vector Norms, Chapman and Hall, London, 1996, pp. 45-90.

13. CASAVOLA, A., E. MOSCA, P. LAMPARIELLO, Robust Ripple-free Deadbeat Control Design, Intl. Journal of Control, 72(6), 1999, pp. 564-573.
14. DORATO, P., Short Time Stability in Linear Time-varying Systems, in: Proceedings of the IRE International Convention Record, New York, 1961.

15. DU, H., C. QIAN, M. T. FRYE, S. LI, Global Finite-time Stabilisation using Bounded Feedback for a Class of Nonlinear Systems, IET Control Theory \& Applications, 6(14), 2012, pp. 2326-2336.

16. EMAMI, A., G. F. FRANKLIN, Dead-beat Control and Tracking of Discrete-time Systems, IEEE Transactions on Automatic Control, 27(1), 1982, pp. 176-181.

17. HADDAD, W. M., A. L'AFFLITTO, Finitetime Stabilization and Optimal Feedback Control, IEEE Trans. Automatic Control, 61(4), 2016, pp. 1069-1074

18. HONG, Y., Finite-time Stabilization and Stabilizability of a Class of Controllable Systems, Systems \& Control Letters, 46(2), 2002, pp. 231-236.

19. HONG, Y., J. HUANG, Y. XU, On an Output Feedback Finite-time Stabilization Problem, IEEE Trans. on Automatic Control, 46(2), 2001, pp. 305-309.

20. HONG, Y., Z. P. JIANG, Finite-Time Stabilization of Nonlinear Systems with Parametric and Dynamic Uncertainties, IEEE Transactions on Automatic Control, 51(12), 2006, pp. 1950-1956.

21. KHALIL, H. K., Nonlinear Systems, third ed., Prentice Hall, New Jersey, 2002.

22. KOTELYANSKI, D. M., Some Properties of Matrices with Positive Elements, Matematicheskii, 31(73), 1961, pp. 961-979.

23. LIU, H., X. ZHAO, H. ZHANG, New Approaches to Finite-time Stability and Stabilization for Nonlinear Systems, Neurocomputing, 138(22), 2014, pp. 218-228.

24. LUR'E, A. I., V. N. POSTNIKOV, On the Theory of Stability of Control Systems, Applied Mathematics and Mechanics, 8(3), 1944, pp. 246-248.

25. MOULAY, E. W. PERRUQUETTI, Finite Time Stability and Stabilization of a Class of Continuous Systems, Journal of Mathematical Analysis and Applications, 323(2), 2006, pp. 1430-1443. 
26. NERSESOV, S. G., W. M. HADDAD, Q. HUI, Finite-time Stabilization of Nonlinear Dynamical Systems via Control Vector Lyapunov Functions, Journal of the Franklin Institute, 345(7), 2008, pp. 819-837.

27. POLYAKOV, A., D. EFIMOV, W. PERRUQUETTI, Finite-time Stabilization using Implicit Lyapunov Function Technique, in: Proceedings of the 9th IFAC Symposium on Nonlinear Control Systems (IFAC Nolcos 2013), Toulouse, 2013.

28. POPOV, V. M., Absolute Stability of Nonlinear Systems of Automatic Control, Automatikai Telemekhanika, 22(8), 1961, pp. 961-979.

29. SERAJI, H., Deadbeat Control of Discrete-time Systems using Output Feedback, International Journal of Control, 21(2), 1975, pp. 213-223.

30. SFAIHI, B., M. BENREJEB, On Stability Analysis of Nonlinear Discrete Singularly Perturbed T-S Fuzzy Models, International Journal of Dynamics and Control, 1(1), 2013, pp. 20-31.

31. STREJC, V., State Space Approach to Linear Computer Control, in: Proceedings of the 7th IFAC/IFIP/IMACS Conf. on Digital Computer Applications to Process Control, Vienna, 1985.

32. SU, Y., C. ZHENG, Robust Finite-time Output Feedback Control of Perturbed Double Integrator, Automatica, 60, 2015, pp. 86-91.
33. TABATABAEIPOUR, S. M., M. BLANKE, Compositional Finite-time Stability Analysis of Nonlinear Systems, in: Proc. of the 2014 American Control Conference (ACC 2014), Portland, 2014.

34. WANG, H., Y. TIAN, C. VASSEUR, NonAffine Nonlinear Systems Adaptive Optimal Trajectory Tracking Controller Design and Application, Studies in Informatics and Control, vol. 24(1), 2015, pp. 05-12.

35. WEISS, L., E. F. INFANTE, Finite-time Stability under Perturbing Forces and on Product Spaces, IEEE Trans. Automatic Control, 12(1), 1967, pp. 341-350.

36. ZHA, W., J. ZHAI, S. FEI, Y. WANG, Finite-time Stabilization for a Class of Stochastic Nonlinear Systems via Output Feedback, ISA Transactions, 53(3), 2014, pp. 709-716.

37. ZHANG, Z. Z. ZHANG, H. ZHANG, B. ZHENG, H. R. KARIM, Finite-time Stability Analysis and Stabilization for Linear Discrete-time System with Timevarying Delay, Journal of the Franklin Institute, 351(6), 2014, pp. 3457-3476.

38. ZHU, L., Y. SHEN, C. LI, Finite-time Control of Discrete-time Systems with Time-varying Exogenous Disturbance, Com. in Nonlinear Science and Numerical Simulation, 14(2), 2009, pp. 361-370. 